\title{
Programa "PERCEP" para Controle Experimental de Pesquisa em Julgamento Temporal em Humanos
}

\author{
"PERCEP" Software for Experiment Control of Temporal Judgment \\ Research with Humans
}

\author{
Valéria Catelli Infantozzi Costa ${ }^{*}$, Eldereis de Paula, \\ José Lino Oliveira Bueno \& Gilberto Fernando Xavier \\ Universidade de São Paulo, Ribeirão Preto, Brasil
}

\begin{abstract}
Resumo
Um programa de computador, o "PERCEP”, foi desenvolvido para a realização dos procedimentos experimentais de estimativa verbal, produção e reprodução temporal em seres humanos. Além da coleta de dados, o programa permite ao experimentador manipular diferentes parâmetros das tarefas e avaliar o impacto dessas manipulações sobre sua aquisição e desempenho. Uma vez que a configuração do programa "PERCEP" é definida pelo próprio experimentador, pode-se realizar uma ampla gama de experimentos acerca dos processos subjacentes ao julgamento temporal. O objetivo do presente trabalho é descrever este programa.

Palavras-chave: Software; julgamento temporal; estimativa verbal; reprodução temporal; produção temporal.

Abstract

A computer program - "PERCEP" - was developed for accomplishing the experimental procedures of verbal estimation, time production and reproduction in human beings. Besides the collection of data, the program allows the researcher to manipulate different parameters of tasks and to evaluate the impact of those manipulations on acquisition and performance. Once the configuration of the "PERCEP" program is defined by the researcher, a wide range of experiments concerning time judgment processing can take place. The aim of the present work is to describe this program.

Keywords: Software; time judgment; verbal estimation; time reproduction; time production.
\end{abstract}

A capacidade de humanos julgarem intervalos temporais pode ser estudada por meio de diferentes procedimentos experimentais básicos, entre eles a estimativa verbal, a produção temporal, e a reprodução temporal (Lalonde \& Hannequin, 1999). Na tarefa de estimativa verbal, apresenta-se ao participante um intervalo de determinada duração, cabendo-lhe indicar a aludida duração (Lalonde \& Hannequin, 1999; Paule et al., 1999). Em uma produção temporal, oralmente ou por escrito, indica-se a duração de um intervalo e se pede que o participante reproduza concretamente tal intervalo, indicando seu início e seu término (Lalonde \& Hannequin, 1999; Paule et al., 1999). No procedimento de reprodução temporal apresenta-se ao participante um intervalo de determinada duração, cabendo-lhe reproduzir concretamente tal intervalo, indicando seu início e seu término (Lalonde \& Hannequin, 1999; Paule et al., 1999).

\footnotetext{
* Endereço para correspondência: Universidade de São Paulo, Faculdade de Filosofia Ciências e Letras de Ribeirão Preto, Departamento de Psicologia e Educação, Av. dos Bandeirantes, 3900, Ribeirão Preto, SP, 14040-901. Tel.: (55) 163602 3697; Fax: (55) 1636335668. E-mail: costaval@ terra.com.br ${ }^{1}$ Agradecimentos: Valéria C. I. Costa é bolsista de Pós-Doutorado da Fundação de Amparo à Pesquisa do Estado de São Paulo (FAPESP), José Lino O. Bueno e Gilberto F. Xavier
}

As tarefas de estimativa verbal e reprodução temporal podem ser realizadas utilizando-se um esquema experimental prospectivo ou retrospectivo. Em um esquema experimental prospectivo os participantes sabem antecipadamente que deverão fazer um julgamento temporal; no esquema experimental retrospectivo os participantes não são informados que um julgamento temporal será necessário até que o evento tenha terminado (Boltz, 1995; Brown, 1985; Lalonde \& Hannequin, 1999). Em parte, a distinção entre estes dois esquemas refere-se ao tipo de instrução que é dada aos participantes durante o contexto experimental; contudo, esta diferenciação de procedimento também se refere a diferentes processos cognitivos subjacentes às respostas dos participantes (Boltz, 1995; Brown, 1985; Lalonde \& Hannequin, 1999). Em um esquema experimental prospectivo os participantes sabem antecipadamente que deverão fazer um julgamento temporal, assim, assume-se que as estimativas realizadas refletem uma duração experimentada ou uma duração em andamento. No esquema experimental retrospectivo os participantes não são infor-

recebem apoio financeiro Pesquisador I do Conselho Nacional de Desenvolvimento Científico e Tecnológico (CNPq). Cópia do programa "PERCEP" e orientação sobre seu uso poderão ser obtidos gratuitamente no site http:// www.brnuede.com/usp/. 
mados que um julgamento temporal será necessário até que o evento tenha terminado. Neste caso, as estimativas ocorrem de modo incidental, ou seja, sobrevêm ao evento cuja duração deve ser estimada, portanto, assume-se que este procedimento permite o acesso à lembrança da duração do evento (Boltz, 1995; Brown, 1985; Pouthas \& Perbal, 2004; Zakay \& Block, 2004).

Uma questão fundamental no estudo do processamento de informações temporais refere-se ao envolvimento de diferentes processos cognitivos, dependendo (a) do esquema do julgamento temporal - prospectivo ou retrospectivo - ou (b) do método pelo qual a resposta é fornecida - reprodução, estimativa ou produção temporal.

Para facilitar a coleta e o tratamento preciso de tais variáveis, desenvolveu-se um programa de computador denominado "PERCEP". Este programa comporta a realização dos procedimentos experimentais de estimativa verbal, produção e reprodução temporal em seres humanos. Além da coleta de dados, o programa permite ao experimentador manipular diferentes parâmetros das tarefas e avaliar o impacto dessas manipulações sobre sua aquisição e desempenho. Uma vez que a configuração do programa "PERCEP" é definida pelo próprio experimentador, podese realizar uma ampla gama de experimentos acerca dos processos subjacentes ao julgamento temporal. O objetivo do presente trabalho é descrever detalhadamente as diferentes possibilidades de configurações do programa, além dos aspectos funcionais de sua interface com o usuário.

\section{Menu de Configurações}

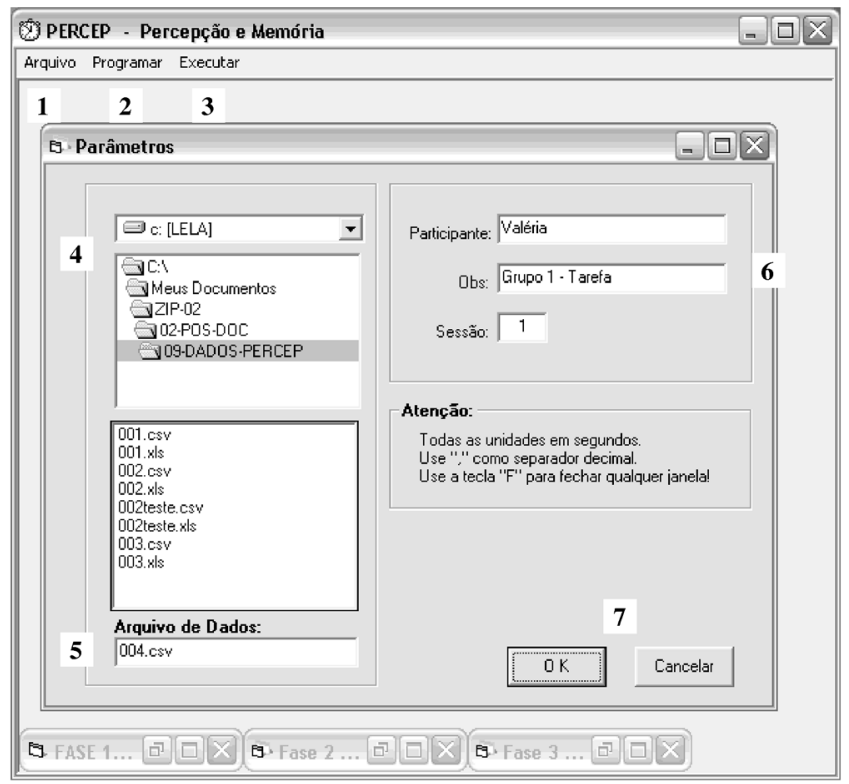

Figura 1. Menu inicial de configurações do programa "PERCEP"; os números indicam itens cuja função é detalhada no corpo do texto

Na Figura 1 é apresentado o menu inicial de configurações do programa "PERCEP". A função de cada campo do menu é detalhada a seguir.
1. Arquivo: este item do menu possui duas opções: (a) Parâmetros - abre a tela do menu Parâmetros e (b) Sair encerra o programa e fecha as telas.

2. Programar: permite a abertura das telas de configuração das Fases 1, 2 e 3.

3. Executar: inicia a execução do programa para a realização do experimento.

4. Pasta de Trabalho: determina-se o local do microcomputador onde serão gravados os arquivos contendo os dados coletados.

5. Arquivo de Dados: determina-se o nome do arquivo no qual serão armazenados os dados de cada participante. A extensão do arquivo deverá ser “.csv”.

6. Identificação: este campo permite incluir o nome do participante e observações.

7. Botões $O K$ e Cancelar: o botão "Cancelar" fecha a respectiva tela; o botão "Ok" registra e armazena as configurações determinadas.

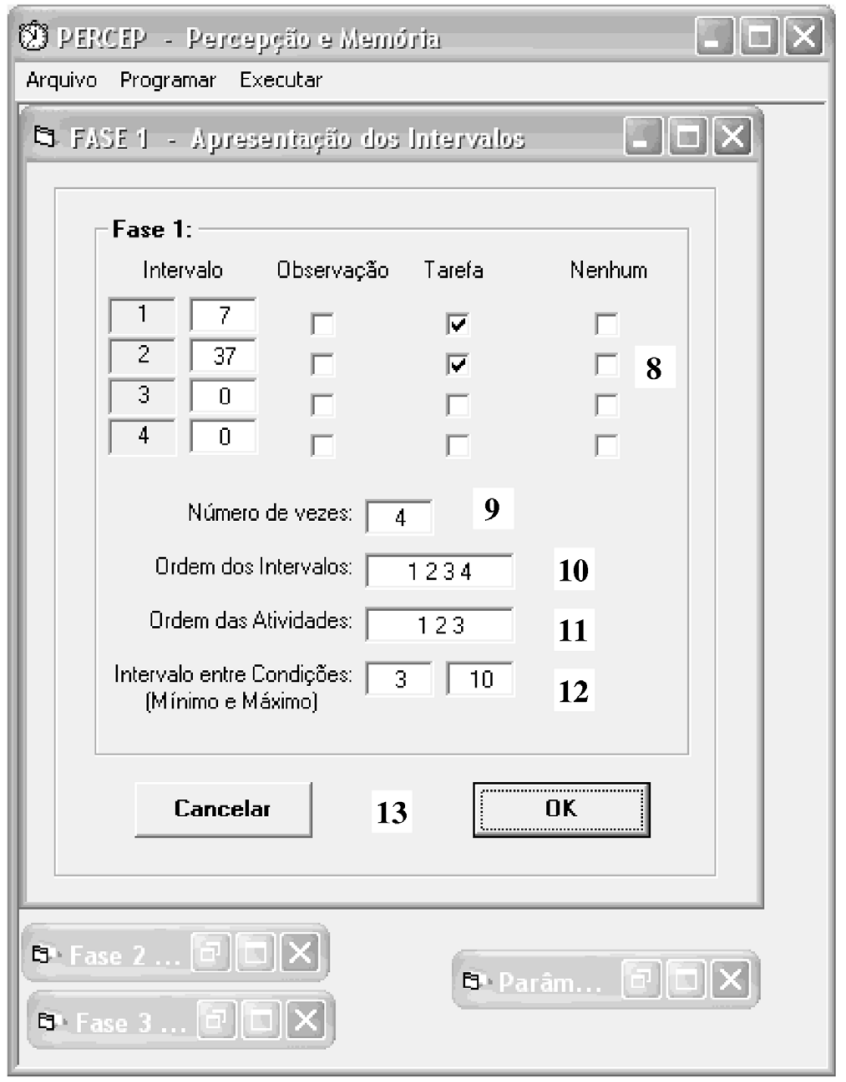

Figura 2. Tela de configurações da Fase 1 - Apresentação dos Intervalos - do programa "PERCEP"; os números indicam itens cuja função é detalhada no corpo do texto

Na Figura 2 é apresentada a tela de configurações da FASE 1 - Apresentação dos Intervalos. Esta fase corresponde à apresentação dos estímulos temporais, isto é, das durações que serão posteriormente julgadas. A função de cada campo do menu é detalhada a seguir.

8. Fase 1: são definidas em quais condições os estímulos temporais serão apresentados; uma condição é definida pelo valor de tempo de um intervalo e a atividade que deve ser 
realizada durante esse intervalo. Neste programa é possível a apresentação de até 4 diferentes intervalos (durante a execução do experimento, o início e final de cada intervalo é indicado pelo som de um beep) e cada intervalo pode ser apresentado em até 3 condições de atividade do participante ("Observação", "Tarefa" e "Nenhum"). Nas colunas "Observação", "Tarefa" e "Nenhum" pode-se determinar se o participante realizará uma tarefa durante a apresentação dos intervalos ("Tarefa"), se ele somente fará a observação de algo, como, por exemplo, a observação de uma figura ("Observação") ou, ainda, se ele não realizará qualquer atividade ("Nenhum"). A execução de uma tarefa é importante especialmente para o esquema Retrospectivo. Neste caso, o participante é instado a realizar tal tarefa quando solicitado pelo programa; posteriormente pede-se que ele reproduza, estime e/ou produza a duração da realização da tarefa. Na seção "Estudo comparativo dos esquemas prospectivo e retrospectivo de julgamento temporal" está descrita a tarefa de 'pintura da estrela', que é um exemplo de tarefa que pode ser utilizada.

9. Número de vezes: define-se quantas vezes cada condição será apresentada, ou seja, quantas práticas serão realizadas para cada intervalo/atividade.

10. Ordem dos Intervalos: determina-se a ordem segundo a qual os intervalos serão apresentados para cada participante. Nesse programa os intervalos não são apresentados aleatoriamente. Para se controlar a variável referente à ordem de apresentação dos intervalos é possível apresentar uma ordem de intervalos diferente para cada participante de um grupo, evitando-se, assim, que a ordem dos intervalos torne-se uma variável interveniente. Por outro lado, com esse programa pode-se estudar o efeito da ordem de apresentação dos intervalos no julgamento temporal ${ }^{2}$.

11. Ordem das Atividades: É possível apresentar os intervalos temporais para cada participante enquanto ele realiza diferentes atividades (ver item 8); assim, este item de configuração permite determinar a seqüência das atividades a qual os participantes deverão realizar para cada intervalo. Ou seja, pode-se apresentar um determinado intervalo em até três condições de atividades, na ordem que o experimentador determinar. Por exemplo, uma primeira vez com a instrução "TAREFA", que aparecerá na tela do computador, a segunda vez, com a instrução "OBSERVAÇÃO”, e ainda, uma terceira vez sem qualquer instrução de realização de atividade. A ordem das atividades, tal como a ordem da apresentação dos intervalos, pode ser uma variável controlada, seja para evitar efeitos espúrios ou como uma variável a ser estudada, dependendo do delineamento experimental realizado. Assim, pode-se estudar se a sequiên-

${ }^{2}$ Pode-se tornar a ordem de apresentação dos intervalos uma variável independente; para tal, basta constituir grupos experimentais de tal sorte que a ordem de apresentação dos intervalos seja a mesma para cada participante de um grupo e diferente para cada grupo. Com a comparação desses grupos, pode-se avaliar se a ordem de apresentação dos intervalos interfere no julgamento temporal dos participantes. cia de atividades interfere no julgamento de um determinado intervalo ${ }^{3}$.

12. Intervalo entre Condições (Mínimo e Máximo): define-se a duração do período entre as apresentações das condições.

\section{Botões $O K$ e Cancelar: ver item 7.}

Na Figura 3 é apresentada a tela de configurações da FASE 2 - Reprodução e Estimativa. Esta fase corresponde à etapa na qual requisita-se aos participantes que façam o julgamento temporal acerca da duração das condições (intervalo/atividade) previamente apresentadas (Fase 1). A função de cada campo do menu é detalhada a seguir.

14. Fase 2: ver item 8. Neste caso são definidas as durações que serão estimadas e se o participante reproduzirá as durações das condições previamente apresentadas realizando uma TAREFA, apenas fazendo uma OBSERVAÇÃO ou fazendo nada. Este campo de configuração permite que se realize um experimento no qual o participante reproduz a duração de uma condição realizando uma atividade diferente da que ele realizou durante a Fase 1 (apresentação). Ou seja, se na Fase 1, um determinado intervalo é apresentado enquanto o participante simplesmente observa uma figura, na fase de reprodução pode-se pedir a ele que reproduza o intervalo apresentado, só que, agora, realizando, concomitantemente, uma dada tarefa. Assim, pode-se estudar os efeitos das condições de apresentação e reprodução dos estímulos, com ou sem atividades.

15. Número de vezes; 16. Ordem dos Intervalos; 17. Ordem das Atividades: estes campos são equivalentes aos campos 9, 10 e 11descritos na Fase 1 (Figura 2). Neste caso, as configurações referem-se aos parâmetros que serão utilizados na Reprodução temporal.

18. Botões OK e Cancelar: ver item 7.

19. Reproduzir os Intervalos e Perguntar valores dos Intervalos: determina-se quais procedimentos experimentais serão realizados - Reprodução, Estimativa, ou ambos. A tela de configuração da FASE 3 - Produção - é idêntica à tela de configuração da FASE 2 (Figura 3) com exceção da ausência do item 19. Nesta tela, as configurações referem-se aos parâmetros que serão utilizados na Produção temporal.

\section{Programa PERCEP em Execução}

Antes de iniciar o experimento, os participantes devem receber instruções referentes à FASE 1 (Apresentação dos estímulos temporais) dependendo dos parâmetros determinados para essa fase. $O$ participante deve ser informado que, quando ouvir um beep, aparecerá na tela do computador a palavra TAREFA ou OBSERVAÇÃO, dependendo do que foi determinado pelo pesquisador. Quando isso ocor-
${ }^{3}$ Pode-se estudar o efeito da seqüência das atividades (tarefa ou observação) no julgamento temporal de determinados intervalos. Esse tipo de procedimento é interessante, em especial, para os estudos relativos à interferência pró-ativa e/ou efeito de primazia/recentidade (primacy/recency effect). 


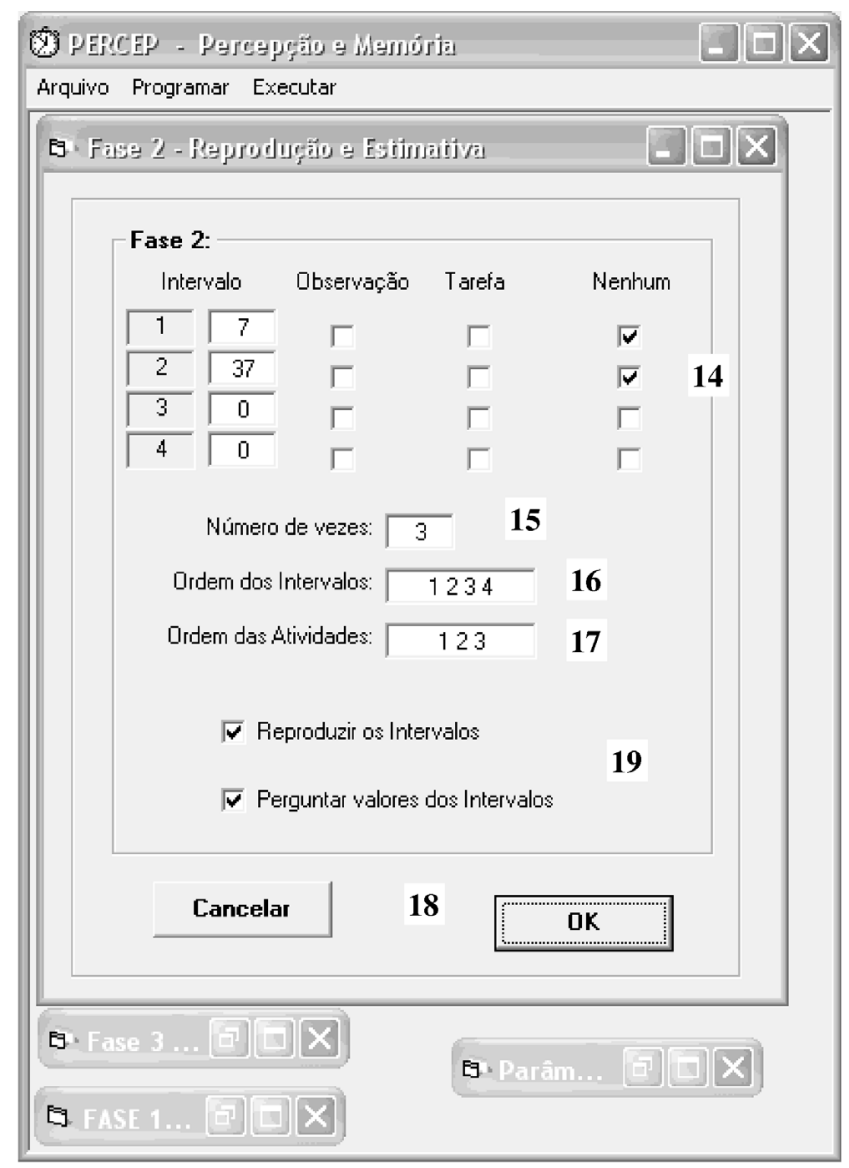

Figura 3. Tela de configurações da Fase 2 - Reprodução e Estimativa - do programa "PERCEP"; os números indicam itens cuja função é detalhada no corpo do texto

rer, o participante deverá realizar a atividade determinada que lhe foi previamente indicada pelo pesquisador. Além disso, o participante é instruído a parar sua atividade e se preparar para as próximas instruções quando, na tela do computador, aparecer escrito "DESCANSE".

Os participantes de um esquema Prospectivo devem ser informados que durante a execução do experimento o início e final de cada intervalo é indicado pelo som de um beep e que eles devem prestar atenção a esses intervalos, pois ao final da FASE 1 eles deverão responder perguntas sobre a duração desses intervalos. A primeira tela que é apresentada aos participantes informa tratar-se da FASE 1 e que eles devem pressionar a tecla "i" para iniciar a fase. Durante a apresentação, reprodução ou produção de cada condição (intervalo/atividade) a tela do computador mostra qual a fase em vigor $(1,2$ ou 3$)$, qual o número da prática (ver item 9) e a instrução "TAREFA", "OBSERVAÇÃO", ou não aparece instrução alguma. Durante os intervalos entre as condições (ver item 12), aparece a palavra "DESCANSE" na tela do computador. Na transição entre uma fase e outra, apresenta-se uma mensagem na tela informando que a fase anterior foi concluída e que será iniciada a fase seguinte, para o que é necessário pressionar a tecla "i". Além disso, nesta tela, aparece escrito "INSTRUÇÕES".
Durante a Reprodução e Produção temporal, após cada intervalo entre as condições, ou seja, no início de uma condição, aparece na tela a instrução para que o participante pressione a barra de espaço para iniciar a contagem de tempo. Após teclar a barra de espaço, a contagem do tempo inicia-se; é informado na tela "O TEMPO ESTÁ CORRENDO" e que o participante deve pressionar a barra de espaço para finalizar a contagem do tempo. Terminada a reprodução temporal de uma condição apresenta-se na tela a pergunta "Quantos segundos você acha que durava o intervalo [número do intervalo; ver item 8]". Abaixo desse texto encontra-se uma caixa de diálogo para o participante digitar o valor que desejar.

Além do que já foi citado, durante o procedimento experimental de Produção temporal, aparece na tela do computador a duração, em segundos, do intervalo que deve ser produzido. Concluídas as fases pré-definidas nas configurações, o programa "PERCEP" apresenta uma tela informando que o experimento foi concluído e agradecendo a colaboração do participante.

O programa "PERCEP" armazena os dados coletados em um arquivo do tipo *.csv. Um exemplo das informações coligidas e armazenadas em um arquivo gerado pelo programa "PERCEP" pode ser visto na Figura 4.

\begin{tabular}{|c|c|c|c|c|}
\hline \# Percep & & & & \\
\hline \# Data $=05-04-20$ & & & & \\
\hline \# Participante $=V_{a}$ & léria & & & \\
\hline$\#$ Sessao = 1 & & & & \\
\hline \# Obs= GRUPO 1 & & & & \\
\hline \# Repetir Fase 1= & & & & \\
\hline \# Repetir Fase 2= & 3 & & & \\
\hline \# Repetir Fase 3= & & & & \\
\hline \# Ordem intervalo & s Fase $1=1,2,3,4$ & & & \\
\hline \# Ordem intervalo & s Fase $2=1,2,3,4$ & & & \\
\hline \# Ordem intervalo & s Fase $3=1,2,3,4$ & & & \\
\hline \# Ordem tarefas $\mathrm{f}$ & ase $1=1,2$ & & & \\
\hline \# Ordem tarefas $\mathrm{f}$ & ase $2=1,2$ & & & \\
\hline \# Ordem tarefas $\mathrm{f}$ & ase $3=1,2$ & & & \\
\hline \# Intervalo condic & oo minimo/maximo Fase 1 & $1=3,10$ & & \\
\hline \# Intervalo condic & oo minimo/maximo Fase 2 & $=0,0$ & & \\
\hline \# Intervalo condic & ao minimo/maximo Fase 3 & $B=0,0$ & & \\
\hline \# Intervalos Fase & $1=7,37,0,0$ & & & \\
\hline \# Intervalos Fase & $2=7,37,0,0$ & & & \\
\hline \# Intervalos Fase & $B=7,37,0,0$ & & & \\
\hline \# Observacao Fas & 1= False, False, False, & False & & \\
\hline \# Observacao Fas & $2=$ False, False, False, & False & & \\
\hline \# Observacao Fas & $3=$ False, False, False, & False & & \\
\hline \# Tarefa Fase $1=$ & True, True, False, False & & & \\
\hline \# Tarefa Fase 2= & False, False, False, False & & & \\
\hline \# Tarefa Fase 3= & False, False, False, False & & & \\
\hline \# Nenhum Fase 1 & $=$ False, False, False, Fals & & & \\
\hline \# Nenhum Fase 2 & $=$ True, True, False, False & & & \\
\hline \# Nenhum Fase 3 & $=$ True, True, False, False & & & \\
\hline \# & & & & \\
\hline Intervalo_medido & Intervalo_questionado & Fase & Numero_Intervalo & Intervalo_real \\
\hline 10.235 & 0 & 2 & 1 & -7 \\
\hline 9.766 & 0 & 2 & 1 & 7 \\
\hline 10.531 & 0 & 2 & 1 & 7 \\
\hline 0 & 7 & 2 & 1 & 7 \\
\hline 27.282 & 0 & 2 & 2 & 37 \\
\hline 31.172 & 0 & 2 & 2 & 37 \\
\hline 32.547 & 0 & 2 & 2 & 37 \\
\hline 0 & 37 & 2 & 2 & 37 \\
\hline 7.875 & 0 & 3 & 1 & 7 \\
\hline 8.922 & 0 & 3 & 1 & 7 \\
\hline 9.813 & 0 & 3 & 1 & 7 \\
\hline 20.204 & 0 & 3 & 2 & 37 \\
\hline 27.782 & 0 & 3 & 2 & 37 \\
\hline 46.954 & 0 & 3 & 2 & 37 \\
\hline
\end{tabular}

Figura 4. Informações coletadas e armazenas no arquivo *.csv para cada participante submetido ao experimento de julgamento temporal realizado pelo programa "PERCEP". Este tipo de arquivo pode ser aberto no aplicativo Microsoft Excel e gravado como arquivo do excel $\left({ }^{*} . x l s\right)$ para posterior análise dos dados 


\section{Estudo Comparativo dos Esquemas Prospectivo e Retrospectivo em Julgamento Temporal}

O programa "PERCEP" está sendo utilizado na coleta de dados de um estudo sobre julgamento temporal que estamos desenvolvendo (Projeto intitulado "Processamento de informações temporais e memória em humanos", FAPESP). A seguir apresentamos resultados parciais deste estudo que ilustram a utilização do programa "PERCEP".

Neste experimento comparou-se a acuidade de julgamentos temporais de estudantes universitários sadios submetidos aos diferentes (a) esquemas - Prospectivo e Retrospectivo - e (b) métodos pelos quais a resposta é fornecida Reprodução, Estimativa verbal e Produção temporal.

Cada participante realizou julgamentos temporais por meio de cada um dos métodos de avaliação para os intervalos de 7 e 37 segundos. Estes intervalos foram apresentados durante a atividade "TAREFA" definida no programa, sendo que, neste caso, a tarefa consistia em pintar com um lápis, dentro do espaço entre as linhas de uma estrela impressa em uma folha de papel. Assim, os intervalos de 7 e 37 segundos foram apresentados aos participantes durante a realização da tarefa de "pintura da estrela". Cada um dos dois intervalos foi apresentado somente uma vez, ou seja, uma prática (Estrela - 1) para um grupo de participantes. Para um segundo grupo, cada intervalo foi apresentado quatro vezes (Estrela - 4). Dois grupos de participantes foram submetidos a tais condições em um esquema Prospectivo, outros dois grupos em um esquema Retrospectivo. Os grupos eram equivalentes em idade, sexo e escolaridade.

A duração (em segundos) dos julgamentos temporais constituiu-se em uma variável dependente que foi medida em diferentes condições: (a) nos métodos de reprodução, estimativa verbal e produção, (b) para um intervalo modelo de $7 \mathrm{~s}$ de outro de 37 segundos, (c) em um esquema prospectivo ou em um esquema retrospectivo, (d) com 4 práticas de apresentação do intervalo temporal ou apenas uma prática. Note que um mesmo participante realiza julgamento temporal em todos os 3 métodos, nos dois intervalos, mas somente em um dos esquemas e com somente um número de práticas. Assim, os participantes foram agrupados em 4 grupos experimentais: Prospectivo - 1 prática $(N=18)$, Prospectivo -4 práticas $(N=15)$, Retrospectivo 1 prática $(N=17)$, Retrospectivo -4 praticas $(N=14)$, sendo que todos os participantes de todos os grupos realizaram julgamentos temporais em cada um dos três métodos (reprodução, estimativa e produção) e para os dois intervalos modelos (7 e 37 s).

A partir da duração dos julgamentos temporais realizados em cada um dos esquemas e condições experimentais calculou-se, para cada participante, o Índice de Eficiência ${ }^{4}$

${ }^{4}$ A duração julgada é subtraída da duração real (7 ou 37 s) ignorando-se o sinal. Este valor representa o erro absoluto, ou seja, o quanto o julgamento afasta-se do valor real, independente do erro ser para mais ou para menos. O erro absoluto é transformado em porcentagem pela divisão deste valor pela
(IE \%), o qual reflete a acuidade temporal, para cada participante. Foram realizadas três ANOVAS distintas, uma, tomando-se os IE \% obtidos a partir da reprodução, outra ANOVA com os valores obtidos na estimativa verbal e a última ANOVA com os IE \% apresentados na produção. Neste caso, utilizou-se "grupo" (1, 2, 3 e 4) como fator inter-sujeito (between) e "intervalo" (7 e 37 segundos) como fator intra-sujeito (within). O teste de Newman-Keuls, complementar a ANOVA, nos permitiu comparar o efeito das variáveis "esquema" e "número de práticas".

Na Figura 5 são apresentadas as médias $( \pm \mathrm{EP})$ dos IE (\%) dos 4 grupos experimentais. A análise dos resultados mostrou que: (a) $\mathrm{O}$ esquema Prospectivo promoveu uma maior acuidade (eficiência) na Reprodução temporal em relação ao esquema Retrospectivo (efeito de Grupo: $\left.F_{1,62}=7,60 ; p<0,01\right)$. Este efeito não ocorreu na Estimativa verbal e Produção temporal (ver indicação $R$ na Figura 5). (b) As reproduções (efeito de Intervalo: $F_{1,60}=21,23 ; p<0,01$ ) e produções (efeito de Intervalo: $F_{1,60}=20,52 ; p<0,01$ ) foram mais acuradas no intervalo de $7 \mathrm{~s}$ do que no intervalo de $37 \mathrm{~s}$. A Estimativa verbal não foi influenciada pela duração do intervalo (ver indicação $7 s$ na Figura 5). (c) A apresentação do estímulo temporal (intervalo) uma ou quatro vezes produziu diferentes efeitos dependendo do esquema e método utilizados.

As condições de apresentação do estímulo temporal não produziram diferenças da acuidade nas estimativas verbais e produções temporais, tanto no esquema Prospectivo como no Retrospectivo. Por outro lado, houve uma diminuição da acuidade das reproduções temporais no esquema Retrospectivo na condição de apresentação do intervalo por quatro vezes em relação à condição de uma apresentação (efeito de Grupo: $F_{3,60}=5,95 ; p<0,01$ ). A acuidade das reproduções não variou segundo as condições de apresentação do estímulo temporal no esquema Prospectivo (ver indicação $E$ na Figura 5). Na Figura 5 estão assinaladas as comparações estatísticas para cada grupo.

Estes resultados reforçam a idéia da existência de diferentes processos cognitivos envolvidos nos esquemas Prospectivo e Retrospectivo, o que se reflete no julgamento temporal quando os participantes precisam reproduzir um intervalo temporal. Por outro lado, a diferença de processos entre esses dois esquemas não aparece quando os participantes devem realizar uma estimativa verbal. Alguns pesquisadores (por exemplo, Brown \& Stubbs, 1992) propõem que julgamentos temporais prospectivos e retrospectivos envolvem os mesmos processos cognitivos; porém, outros pesquisadores argumentam que diferentes processos cognitivos estão subjacentes a tais julgamentos (Zakay \& Block, 2004). Neste caso, em um procedimento

duração real e multiplicando-se por 100. Este valor indica a porcentagem de erro do participante, ou seja, representa a porcentagem de ineficiência do julgamento temporal realizado (Brown, 1985; Franssen \& Vandierendonck, 2002). Subtraindo-se a porcentagem de erro de 100 , obtém-se a porcentagem de eficiência, sendo este o valor aqui apresentado. Por exemplo, se a porcentagem de erro de um participante é igual a 30\%, o Índice de Eficiência deste participante é de $70 \%$. 

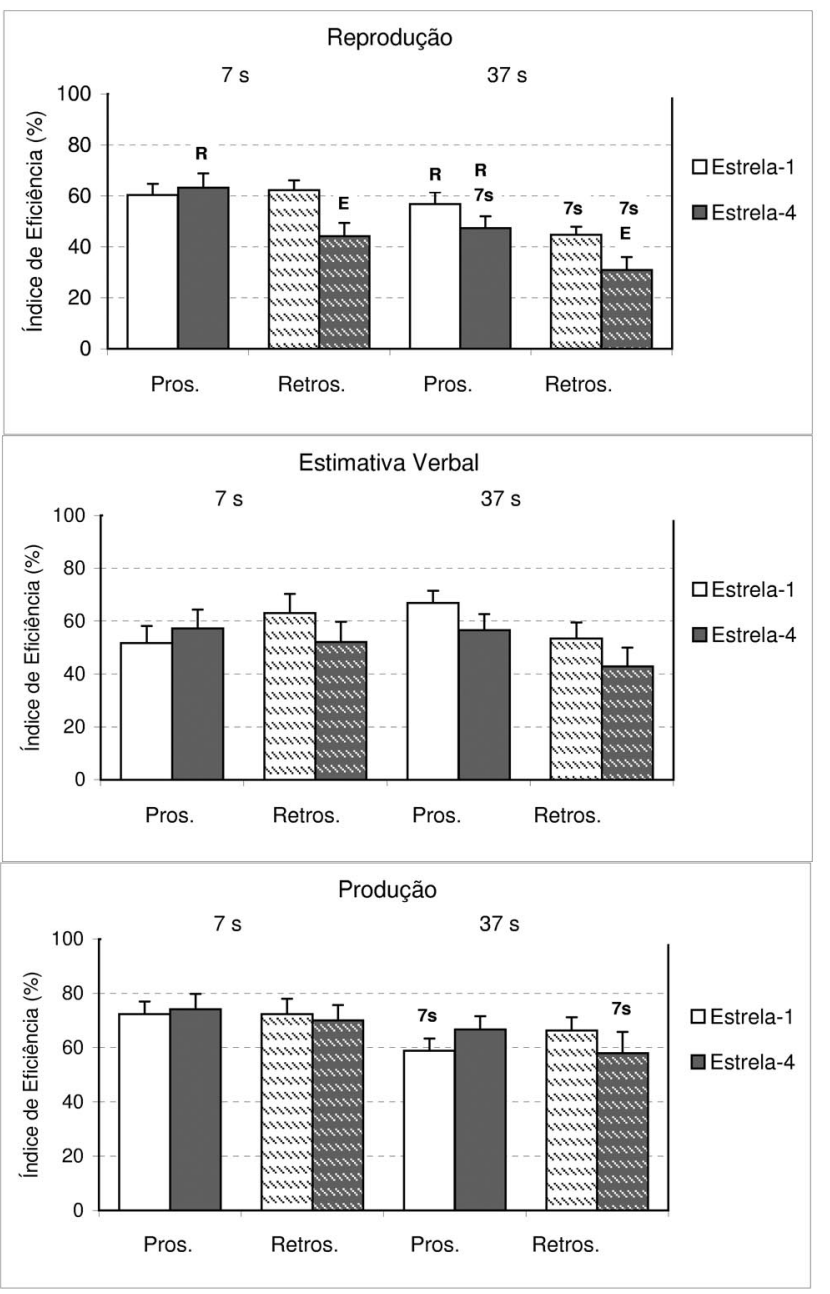

Figura 5. Média ( \pm EP) dos Índices de Eficiência (\%) dos participantes que realizaram a Reprodução, Estimativa verbal e Produção em dois intervalos temporais - 7 e 37 s e em duas condições - cada intervalo é apresentado uma vez (Estrela - 1) e cada intervalo é apresentado 4 vezes (Estrela - 4) nos esquemas Prospectivo (Pros.) e Retrospectivo (Retros.). Hachurada a condição Retrospectiva. (E) diferença significativa em relação ao grupo correspondente submetido a condição Estrela-1; (7 s) diferença significativa em relação ao grupo correspondente submetido ao intervalo de $7 \mathrm{~s}$; diferença significativa em relação ao grupo correspondente submetido ao esquema Retrospectivo. (Teste de Newman-Keuls, $p<0,05$ )

prospectivo, no qual a pessoa está ciente de que fará um julgamento temporal, o processo envolvido dependeria essencialmente da atenção. Por outro lado, a memória seria o processo essencial subjacente à capacidade de julgamento temporal em um procedimento retrospectivo, no qual deve-se lembrar uma determinada duração previamente experimentada. Contudo, resultados inconsistentes têm sido encontrados na literatura, como apontam Zakay e Block (2004) em sua revisão sobre o tema. Esses autores afirmam ser essencial a obtenção de mais resultados que mostrem uma dupla dissociação entre tais procedimentos para que se possa concluir pela distinção de diferentes processos cognitivos subjacentes aos procedimentos prospectivo e retrospectivo de julgamento temporal. Desta forma, os resultados finais do presente estudo, aqui descritos somente como ilustração do funcionamento do programa PERCEP, poderão contribuir para a discussão, ainda em curso, do tema.

Os julgamentos temporais podem decorrer do processamento de informações derivadas da percepção imediata (memória imediata), da memória operacional ou da memória declarativa. Essas informações podem ser dados temporais diretos - por exemplo, os fornecidos pelo relógio - ou conhecimentos temporais coletados de todas as informações percebidas e recordadas. Quando originalmente percebidas e recordadas, as sequiências não foram, necessariamente, tomadas como informações que pudessem produzir julgamentos temporais. O indivíduo também pode, com o propósito de estimar a passagem do tempo, atentar para seus estados fisiológicos tal como sensação de fadiga. As informações não-temporais (seqüência de eventos, estados fisiológicos etc.) das quais podem ser inferidos julgamentos temporais também são conhecidas como pistas ou dicas temporais (informações temporais). Estimativas pouco apuradas conduzem o indivíduo a subestimar a passagem do tempo; quando há poucas pistas (dicas) temporais percebidas ou recordadas, os sujeitos produzirão estimativas com intervalos mais curtos, daí decorrendo a aludida subestimação (Williams, Medwedeff, \& Haban, 1989).

Levando em conta estas idéias apresentadas por Williams et al. (1989), pode-se pensar que em um esquema ou julgamento prospectivo, os sujeitos, durante a apresentação da duração do intervalo (fase de codificação), podem "coletar" o maior número possível de pistas temporais, o que tornaria seus julgamentos mais longos e mais acurados do que os julgamentos retrospectivos. Já os sujeitos sob um esquema retrospectivo de julgamento temporal não teriam a mesma oportunidade; tendo à sua disposição poucas pistas temporais, eles tenderiam a subestimar os intervalos temporais. Entretanto, no esquema retrospectivo, o intervalo a ser julgado é apresentado, em geral, somente uma vez.

Assim, o principal objetivo deste estudo era verificar se proporcionando aos participantes submetidos a um esquema retrospectivo a oportunidade de "coletar" um número maior de pistas temporais, o seu desempenho melhoraria. Para testar esta hipótese foram comparadas a acuidade temporal de participantes submetidos à exposição do estímulo temporal somente por uma vez com a acuidade temporal de participantes submetidos 4 vezes à exposição do estímulo temporal. A apresentação repetida de um intervalo poderia levar a um julgamento temporal mais acurado na medida em que a maior exposição ao estímulo possibilitaria a "coleta de um maior número de pistas temporais." Entretanto, os resultados presentes mostraram que, no esquema Prospectivo, a acuidade do julgamento temporal não foi diferente entre os grupos nos quais apresentou-se o intervalo uma só vez ou quatro vezes. Além do mais, no esquema Retrospectivo, um número maior de apresentações dos estímulos temporais diminuiu a acuidade das Reproduções. Ou seja, quando os julgamentos temporais 
refletem uma duração experimentada (Prospectivo), uma ou quatro apresentações de um intervalo promovem uma coleta de pistas temporais equivalentes e não produzem diferenças na acuidade da Reprodução. Por outro lado, quando os julgamentos temporais refletem a lembrança da duração do evento (Retrospectivo) a apresentação de um intervalo por quatro vezes parece causar um efeito de interferência pró-ativa, prejudicando, assim, a acuidade da Reprodução temporal.

Deste modo, estes resultados mostram que o programa "PERCEP" permite a coleta de dados acerca de julgamentos temporais realizados em procedimentos experimentais de estimativa verbal, produção e reprodução temporal, possibilitando o estudo das relações de tais procedimentos com a acuidade (e também com a precisão e super- e sub-estimativas, não apresentadas neste exemplo). Além disso, o programa possibilita a introdução de variáveis, tais como (a) tipo de tarefa realizada durante a apresentação do intervalo temporal, (b) número de vezes em que o intervalo temporal é apresentado, (c) seqüência da apresentação de diferentes intervalos temporais, (d) seqüência das tarefas (ou das atividades) que podem ser desenvolvidas durante a apresentação dos intervalos temporais, (e) interação entre os diferentes procedimentos experimentais (reprodução, estimativa, produção); permitindo dessa forma, estudo de diversos fatores que afetam (ou possam afetar) o julgamento temporal humano.

\section{Referências}

Boltz, M. G. (1995). Effects of event structure on retrospective duration judgments. Perception and Psychophysics, 57(7), 1080-1096.

Brown, S. W. (1985). Time perception and attention: The effects of prospective versus retrospective paradigms and task demands on perceived duration. Perception and_Psychophysics, 38(2), 115-124.

Brown, S. W., \& Stubbs, D. A. (1992). Attention and interference in prospective and retrospective timing. Perception, 21, 545557.

Franssen, V., \& Vandierendonck, A. (2002). Time estimation: Does the reference memory mediate the effect of knowledge of results? Acta Psychologica, 109, 239-267.

Lalonde, R., \& Hannequin, D. (1999). The neurobiological basis of time estimation and temporal order. Reviews in the Neuroscience, 10(2), 151-173.

Paule, M. G., Meck, W. H., Mcmillan, D. E., Mcclure, G. Y., Bateson, M., Popke, E. J., Chelonis, J. J., \& Hinton, S. C. (1999). The use of timing behaviors in animals and humans to detect drug and/or toxicant effects. Neurotoxicology and Teratololy, 21(5), 491-502.

Pouthas, V., \& Perbal, S. (2004). Time perception depends on accurate clock mechanisms as well as unimpaired attention and memory processes. Acta Neurobiologiae Experimentalis, 64(3), 367-385

Williams, J. M., Medwedeff, C. H., \& Haban, G. (1989). Memory disorder and subjective time estimation. Journal of Clinical and Experimental Neuropsychology, 11(5), 713-723.

Zakay, D., \& Block, R. A. (2004). Prospective and retrospective duration judgments: An executive-control perspective. Acta Neurobiologiae Experimentalis, 64(3), 319-328. 